\title{
Le titre de wr mD Smaw le chef des dix de la Haute Egypte
}

\section{Dr. Nevine Hussein Tolba}

Maître de conférence à la facultée de Lettres, l'Université d'Ain Chams nevinetolba@yahoo.com

Le titre en question est très intéressant puisque il avait une grande importance au cours de toutes les époques. Il avait plusieurs écritures et plusieurs traductions.

Ce titre

signifie le grand des dix de la Haute Egypte. Le titulaire était un officier attaché au bureau du vizir. ${ }^{1}$ Le grand nombre de titulaires indique leur position sociale dans le cercle de la cour. ${ }^{2}$ Ce titre peut aussi signifier une organisation de groupe de dix personnes. ${ }^{3}$ Quirke traduit ce titre "le chef des dix de la Haute Egypte".

Ce titre remonte à la préhistoire, à Naqqada II. Il est classifié comme un groupe de dix. Ensuite, les intitulés étaient des hauts fonctionnaires, c'est un réservé aux officiers. Il apparaît avant le nom de la personne portant le titre. De même que sur les blocs du Louvre et des statues. ${ }^{4}$ La Haute Egypte était divisée en des nomes gouvernés chacun par un nomarque dont son administration dépendait. Le grand des dix avait des fonctions administratives dans les nomes. ${ }^{5}$

Ce titre existait durant l'Ancien Empire $n^{2}$, il signifie aussi Magistrat des

${ }^{1}$ Quirke, S. (1986). <<The Regular Titles of The Late Middle Kingdom>>, RdE37, Bouvain, p.120.

${ }^{2}$ Stefanovic, D.( 2009). The Non-Royal Regular Feminine Titles of the Middle Kingdom and Second intermediate Period, GHP, London, p.35;

Helck, W.( 1954). <<Untersuchungen zu den Beamtentiteln des Agyptischen Alten Reiches>>, Agyptologische Forschungen 18, p.19.

${ }^{3}$ Strudwick, N.(1985). The Administration of Egypt in the Old Kingdom, the highest titles and Their Holders, Boston, p.222.

${ }^{4}$ Helck, W.( 1987). <<Untersuchungen zur Thinitenzeit〉>, Wiesbaden, Agyptologische Abhandlungen 45,p.243.

${ }^{5}$ Erman, A.( 1885). Aegypten und Aegyptisches Leben im Altertum,I,p.124.

${ }^{6}$ Jones, D.( 2000). An Index of Ancient Egyptian Titles, Epithets and Phrases of Old Kingdom I, International Series 866, p.388 n 1437. 
dix de la Haute Egypte associés aux pouvoirs du vizir. ${ }^{8}$ Durant la fin du Moyen Empire et de la seconde période intermédiaire réguliers les plus communs ${ }^{9} \mathrm{Il}$ se trouve sur les parois de la tombe de vizir Rekhmirê durant la XVIIIème dynastie. Empire. ${ }^{10}$ Il était abondamment cité dès la fin de la XIIème dynastie ${ }^{11}$. Il devient fréquent à la XIIIème dynastie et la seconde période intermédiaire. ${ }^{12}$

Ce titre est attaché au bureau du vizir selon les pouvoirs du vizir. Donc en général, le grand de dix de la Haute Egypte désigne le personnel du vizir ou les officiers de son bureau. De même, le porteur de ce titre peut avoir servi dans d'autres bureaux nationaux mais les sources l'ont aligné avec le vizir. La présence de ce titre sur les monuments de la fin de la XIIIème dynastie indique qu'il était très répandu durant cette période. Les titulaires étaient nombreux dans la liste des noms du papyrus Boulaq 18 au début de la même dynastie. ${ }^{13}$

Dans les archives du début du Moyen Empire, le titre apparaît à l'extérieur de la vallée du Nil tandis que les sources de la fin du Moyen Empire, placent le titre dans les archives de l'intérieur de la vallée. ${ }^{14}$

Le grand des dix de la Haute Egypte participait aux expéditions d'exploitations des mines et des carrières. Ils étaient des hauts fonctionnaires mais ils n'avaient pas une classe sociale élevée et n'avaient pas un rang supérieur. $^{15}$

${ }^{7}$ Murray,(2004) Index of Names and Titles of The Old Kingdom, New York, p.XIX.

${ }^{8}$ Ibid.,p.197,p.222.

${ }^{9}$ Ward, W. (1982).Index of Egyptian Administrative and Religious Titles in

The Middle Kingdom with a Glossary of Words and Phrases used by

W.Ward, Beirut, p.87 n 721.

${ }^{10}$ Ayedi, A.R.Al.( 2006). Index of Egyptian Administrative Religious and Military Titles of The New Kingdom, Egypt,p.244.

${ }^{11}$ Vernus, P.( 1974).<<Un Pseudo-Naos de la XIII dynastie〉>, RdE 26,Paris, p.111.

${ }_{12}$ Ibid.,p.107.

${ }^{13}$ Quirke, (2004).S. Titles and Bureaux of Egypt, GHP, Egyptology I, London, p.87.

${ }^{14}$ Quirke, STitles and Bureaux of Egypt, p.87.

${ }^{15}$ Vernus, P.( 1974). RdE 26,Paris, p.109. 
Ce titre est aussi traduit $<<$ le grand conseil des dix $>>$. Il constitue le grand conseil du gouvernement de l'Egypte pendant l'Ancien Empire. Les membres de conseil de dix étaient des hauts fonctionnaires. ${ }^{16}$ En plus de ces fonctionnaires qui avaient atteint les plus haut niveaux de la hiérarchie comme gouverneurs de nomes, le conseil des dix renfermait les magistrats qui travaillaient dans l'administration judiciaire. Ce conseil organisait judiciairement le pays, ainsi que la direction de cette administration. Les membres de ce conseil portaient les titres de la direction des services de la justice $<<$ maîtres de l'administration judiciaire $>$, $<<$ directeurs de l'administration judiciaire $>>,<<$ directeurs de la justice $>$, $<<$ directeurs des jugements à l'administration judiciaire $>>$. Des membres de ce conseil étaient sélectionnés peut être les conseillers qui aident les présidents de chambre de la cour suprême. ${ }^{17}$

Parmi ces conseillers on peut mentionner: <<chefs des secrets des jugements $>>,\langle<$ chefs des secrets des instructions secrètes de la grande cour des six $>>,\langle<$ chefs des secrets des instructions secrètes $>>$. Les membres de ce conseil sont les seuls à côté du vizir qui portaient le titre de $<<$ directeur du double service de l'enregistrement $\gg>$ et $<<$ directeur de la maison des impots >. Donc on peut en déduire que le membre du conseil des dix dirigeait ces services. Mais les finances formaient une organisation séparée. A la difference des autres sections importantes comme les travaux publics, la chancellerie, l'administration militaire était dirigée par des fonctionnaires qui n'étaient pas des membres du conseil des dix. Quand l'Egypte était divisée en deux gouvernements, Nord et Sud, les gouverneur de ces deux parties du pays étaient choisis parmi les membres du conseil des dix. ${ }^{18}$

Les membres du conseil des dix sont les plus hauts fonctionnaires de l'administration civile. Le roi choisissait ses conseillers de législation parmi eux. Ils étaient les chefs des secrets du roi, les secrètaires de législation et les chefs des secrets des affaires étrangères. Le conseil des dix était l'organe vital de l'administration et avait un personnel spécial. Chaque membre avait ses bureaux. Chaque membre du conseil dirigeait une partie spécifique de l'administration. ${ }^{19}$

Ils occupaient de hautes fonctions judiciaires celles de conseillers à la haute cour des six. Durant la VI dynastie les membres du conseil des dix

\footnotetext{
${ }^{16}$ Pirenne, J. (1934).Histoire des Institutions et du droit privé de l'ancienne Egypte II, Bruxelles,p.101.

${ }^{17}$ Pirenne, J. op.cit.,p.102.

${ }^{18}$ Pirenne, J. op.cit.,p.103.

${ }^{19}$ Pirenne, J.op.cit.,p.104.
} 
étaient des gouverneurs de nomes et des nomarques héréditaires. ${ }^{20}$ Pourtant à la fin de la VI dynastie, les membres du conseil ne sont plus des personnes principaux et le rôle du conseil diminue jusqu' à ce qu'il perde sa position centrale dans le gouvernement. Ses membres n'étaient plus des conseillers d'audience à la haute cour. Malgré tout, il ne disparaît pas complétement. Le décret de Coptos cite les fonctions administratives du conseil de dix qui dirige le service des déclarations et des impôts. ${ }^{21} \mathrm{Ce}$ conseil fut considéré comme le conseil supérieur de l'administration royale. Mais ce rôle a disparu à la fin du règne de Pepi II. ${ }^{22}$

Les porteurs du titre étaient des personnes qui avaient participé à la fête Sed cité sur la stèle de British Museum 101, ainsi qu'au Nouvel Empire. ${ }^{23}$

Ce titre était antérieurement lu Wr mabAyw Smaw qui signifie le magnat des trente de la Haute Egypte. Il travaillait comme personnel légal du vizir et pour d'autres officiers aussi. ${ }^{24}$ Selon Ward, ce titre se lit Wr mDw Smaw qui veut dire magnat de dix de la Haute Egypte. ${ }^{25}$ Ce titre fut très répandu durant le Moyen Empire, plus de 75 individus sont connus datant de cette période. Il devait exister partout en Egypte. Le sens général doit être un membre du tribunal de droit. $^{26}$

Il y a de nombreux écrits sur le grand des dix de la haute Egypte.
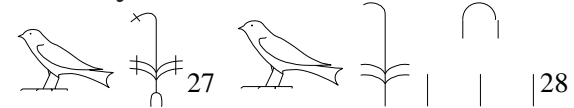

28. Le Magistrat des dix de la Haute Egypte était un titre administratif. ${ }^{29} \mathrm{C}^{\prime}$ était une fonction judiciaire. ${ }^{30}$ Ce titre peut être traduit le grand de trente de la Haute Egypte comme Grapow ${ }^{31}$ et Gardiner. ${ }^{32}$

${ }^{20}$ Pirenne, J.( 1935). Histoire des Institutions et du droit privé de l'ancienne Egypte III, Bruxelles p. 73.

${ }^{21}$ Pirenne, J. Histoire III,p.74.

22 J.Pirenne, Histoire III,p.75.

${ }^{23}$ Vernus, P. (1974).RdE 26,Paris, p.107.

${ }^{24}$ Ward, W.( 1986). Essays on Feminine Titles of the Middle Kingdom, and Related Subjects, Beirut, p.123.

${ }^{25}$ Ward, W. Index of Egyptian Administrative, p.87 n 721.

${ }^{26}$ Ward, W. Essays on Feminine Titles, p.120

${ }^{27} \mathrm{~Wb}$ II, 184,2.

${ }^{28} \mathrm{~Wb}$ I,329(13); Wb IV, 473(15).

${ }^{29}$ Faulkner,R.(1981). A Concise Dictionary of Middle Egyptian, Oxford, p.64, p.123.

${ }^{30}$ Rowe, A. (1939). <<Three new Stelae from the south Eastern Desert >>, ASAE 39, Le Caire, p.192 photenote n 1.

${ }^{31} \mathrm{~Wb}$ I, p.329. 


\section{Des exemples de porteurs du titre:}

Comme les officiers kAi, anX-m-kAi, nTr-wsr, sXm-anx-ptH(,sSm-nfr; $\mathrm{HbA}$, nfr-sSm ptH, Hkni-Xnmw. ${ }^{33}$

Un porteur du titre nommé Nb-swmnw apparaît sur la stèle Vienne 117, stèle du Musée égyptien du Caire CGC 200093 et sur la stèle de Bolton. ${ }^{34}$ Un autre grand des dix de la Haute Egypte $\mathrm{Nb}$-irf est abondamment mentionné à Edfou, il est le propriétaire de la stèle de Louvre E 14330 et de la statue philadelphie E $878 .{ }^{35}$ Un autre porteur du titre portait le nom de Rnsnb. Il a été découvert sur un sceau datant de la XII dynastie. C'est un sceau obsidien. ${ }^{36}$

Nous connaissons plusieurs porteurs du titre comme les deux frères Rnw et iai-ib, les fils de la reine $x$ a-s-nbw datant de la XIII dynastie. ${ }^{37}$ De même que Sahi, fils de la dame Nebitef, figure sur la stèle de Leningrad, provenant d'Abydos, accompagné de son frère. Ainsi que l'embaumeur Nebneb, fils de la dame Noubhersaoues ceci au Moyen Empire. ${ }^{38}$

Un grand des dix de la Haute Egypte nommé Khonsou figure sur la stèle de Wien datant de la XIII dynastie ainsi que la stèle de Seneb du Musée Egyptien du Caire CGC 200093. Les deux étaient des grands des dix. ${ }^{39}$

Quant à Amen-hotep, le père de Siamon, il figure sur une stèle de Thèbes et sa mère est la maitresse de maison Hann. ${ }^{40}$ Ainsi qu'un autre grand des dix

${ }^{32}$ Gardiner, A.H. (1931).The Chester Beatty Papyri, No 1, p.14 photnote 3 ( les trente magistrats); A.Rowe,op.cit.,p.192 photenote 1.

${ }^{33}$ Strudwick N.( 1985).The Administration of Egypt in the Old Kingdom, Boston, p.179 n 36,31,91,124,132,87,99.

${ }^{34}$ Meulenaere, De. ( 1977). <<Review of W.K.Simpson, The Terrae of the Great God at Abydos, The Offering Chapels of Dynasties 12 and 13>>, CdE52 n 103, Bruxelles, p.8.

${ }^{35}$ Meulenaere, H.de.( 1981). <<Contributions à la prosopographie du Moyen Empire >>, supp. BIFAO 81, Le Caire,p.81; Engelach, B. (1922). <<Stelaes and Tables of Offerings of the Late Middle Kingdom from Tell Edfu $>>$, ASAE 22, Le Caire, p.115.

${ }^{36}$ Martin, G.T.( 1979). <<Private-name Seals in the Alnwick Castle Collection >>, MDAIK 35, Meinz, p.218 n 17.

${ }^{37}$ Spalinger, A.( 1980). <<Remarks on the Family of Queen $x$ a $-s-n b w$ and the Problem of Kingship in Dynasty XIII >>, RdE 32, Louvain, p.97. ${ }^{38}$ Walle, B.Van de \& Meulenaere, H.de.( 1973). <<Compléments à la prosopographie médicale >, $R d E$ 25, Paris, p.71.

${ }^{39}$ Franke, D.( 1983). Altagyptische Verwandtsbezeichnungen im Mittleren Reich, Hamburg,p.21.

${ }^{40}$ Hayes, The Scepter of Egypt, New York 1953, p.346. 
est représenté sur une stèle à Ouadi Houdi au désert oriental. C'est MontouHotep fils de Henenou fils de Bebi. ${ }^{41}$ En plus d'Antef fils de Sobek-Rê qui, lui, figure sur une autre stèle à Ouadi Houdi au désert oriental mais malheureusement, il ne subsiste que la partie inférieure. ${ }^{42}$ Ainsi que Sahi fils de la dame Nebitef, il figure sur une stèle provenant d'Abydos, datant du Moyen Empire, conservé au Musée de l'Ermitage à Leningrad. ${ }^{43}$

On trouve également le grand des dix Imny représenté sur un naos dans le temple de HqA-ib à Elephantine datant de la XIII dynastie. ${ }^{44}$ Puis, Imn.mHAt fils de Rn.s-snb qui figure sur la stèle CGC 20520 datant de la fin de la XIIe ou la XIIIIe dynastie. ${ }^{45}$ snb.f-n.i et son père NHy étaient des grands des dix de la Haute Egypte à l'époque de la XIIIe dynastie.. ${ }^{46} \mathrm{Bmbw}$ est représenté sur la stèle de Vatican MG 170 datant de la XIIIe dynastie. ${ }^{47}$ MnTw Htp anX f père de SanX-ib-ra-snb figure sur la stèle Avignon A31 datant de la XIIIe dynastie, les deux étaient des grands des dix de la Haute Egypte. $^{48}$

Le grand des dix de la Haute Egypte existait pendant le Moyen Empire comme cela est mentionné par le papyrus Kahoun. Il servait comme conseiller du vizir. Même durant l'Ancien Empire, il avait des fonctions judiciaires. ${ }^{49}$ C'étaient des hauts fonctionnaires. Ce titre est associé avec le prêtre dans $\mathrm{PH}-\mathrm{r}-\mathrm{nTr}$ et Wp-m-nfrt. Il jouait un rôle important dans la cour royale. Les membres du conseil des dix participaient à la fête Sed. Ce titre fut abandonné à la fin de la VIe dynastie. ${ }^{50}$ Mais sera réutilisé durant le Moyen Empire.

D'autres exemples de personnes appelées Ddw-nSmt dans la région de Gebelin datent de la XIIIe dynastie. ${ }^{51} \mathrm{Ddw}-$ Sbk/bbi figure sur la stèle de Louvre $\mathrm{C} 13$ et $\mathrm{C} 285$ de la XIIIe dynastie. ${ }^{52}$ Ce titre qui était répandu pendant le Moyen Empire, n'avait subi aucun changement dès l'Ancien

${ }^{41}$ Fakhry, A.( 1952). The Inscriptions of the Amethyst Quarries at Wadi El

Hudi, Cairo, p.33 fig. 27.

${ }^{42}$ Ibid.,p.44 n 228 fig.36.

${ }^{43}$ Walle, B.Van de \& Meulenaere, H.de.( 1973). <<Compléments à la

prosopographie médicale $>, R d E$ 25, Paris,p.71.

${ }^{44}$ Vernus, P. (1986). Le surnom au Moyen Empire, Rome, p.12 n 47.

${ }^{45}$ Ibid.,p. 21 n 89.

${ }^{46}$ Vernus, P. Le surnom,p.27 n 114.

${ }^{47}$ Vernus, P. Le surnom,p.37n 160.

${ }^{48}$ Vernus, P. Le surnom,p.56 n 261,p.57 n 261.

${ }^{49}$ Helck, W. (1954). <<Untersuchungen zu den Beamtentiteln des

Agyptischen Alten Reiches>>, Agyptologische Forschungen 18,p.19

${ }^{50}$ Ibid.,p.48.

${ }^{51}$ Vernus, P. Le surnom,p.71 n 323.

${ }^{52}$ Vernus, P. Le surnom,p.72 n 330. 
Empire. Selon Sethe, il ne doit pas être traduit

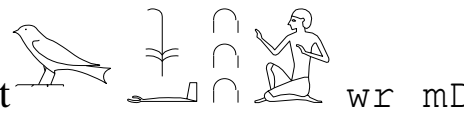
Smaw le grand des 30 de la Haute Egypte comme c'était le cas général, mais ce groupe renferme trois signes de dix qui représentent simplement l'idée de pluralité des dizaines comme une variante. Ce titre doit être traduit "le grand des collèges de dix (magistrats ou fonctionnaires) du Sud. Dans l'inscription de Sahi, on voit une orthographe intermédiaire, le premier signe $\mathrm{mD}$ est combiné avec sw et deux autres signes $\mathrm{mD}$ sont surajoutés à côté du groupe entier. L'orthographe est la même durant l'Ancien et le Moyen Empire. ${ }^{\prime}$

Le grand des dix de la Haute Egypte était répandu pendant le Nouvel Empire. $^{54}$

\section{Les différentes écritures du titre:}

Durant l'Ancien Empire ce titre s'était écrit

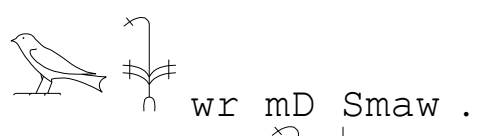

Durant le Moyen Empire

ce titre s'était écrit

ou

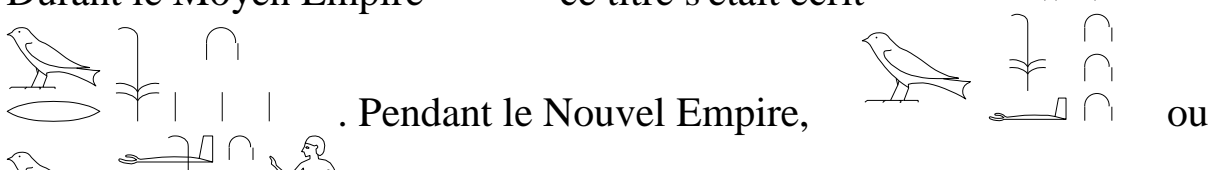

écrit sur la base d'une statue n 1905 provenant de

Thèbes. Il peut être aussi traduit "le grand de dix compagnons de la Haute Egypte". ${ }^{55}$ On distingue le signe Smaw dans le titre de dieu Seth d'Ombos "le maître de la Terre de la Haute Egypte". c'est le nom fréquent du dieu ${ }^{56}$.Le

titre

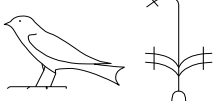

wr mD Smaw signifie Les grands de la Haute et la Basse Egypte cité par Murray dans les Mastabas de Saqqara datant de la IIIe dynastie, durant la VIe dynastie et la XVIIIe dynastie ${ }^{57}$. PHr nfr était intitulé le grand de dix de la Haute Egypte, à cause de sa haute situation, il avait des

${ }^{53}$ Walle, Van de. (1938). <<Un hymne du Moyen Empire complété à l'aide de deux stèles du Musée National de Rio de Janero>>, RdE 3, Le Caire, p.96. ${ }^{54}$ Vernus, P. ( 1974). RdE 26, Paris, p.107.

${ }^{55}$ Sethe, K. ( 1918). $<<\mathrm{Zu}$ den mit wr $>>$ der größe $<<$ beginnenden alten Titeln>>, ZÄS 55, Leipzig, p.66.

${ }^{56}$ K.Sethe,( 1907-8). <<Die Namen von Ober und Unteragypten usw〉>, Z̈̈S 44, Leipzig, p.16.

${ }^{57}$ Sethe, K. (1907-8), ZÄS 44, Leipzig, p.15 
fonctions judiciaires comme juge dans la cour. Son titre est observé sur le linteau d'une fausse porte. ${ }^{58}$

Ce titre avait une grande importance durant toutes les époques pharaoniques liées aux vizirs. Ses membres avaient diverses fonctions ce qui prouve l'importance de ce titre. Toutes les écritures variées que nous avons observées ainsi que les traductions du titre comme "le chef de dix de la Haute Egypte", "le grand de dix compagnons de la Haute Egypte", "Magistrat de dix de la Haute Egypte", "le grand conseil de dix", "le magnat de trente de la Haute Egypte","le grand des 30 de la Haute Egypte". Tout cela traduit le même pouvoir et la même situation durant toutes les époques. A mon avis, Ce titre signifie le chef des dix de la Haute Egypte.

\section{Liste des références et des périodiques:}

- Ayedi, A.R.Al.( 2006). Index of Egyptian Administrative Religious and Military Titles of The New Kingdom, Egypt, p.244.

-Engelach, B. (1922). $<<$ Stelaes and Tables of Offerings of the Late Middle Kingdom from Tell Edfu〉>, Annales du Service des Antiquités Egyptiennes 22, Le Caire, p.115.

-Erman, A.( 1885). Aegypten und Aegyptisches Leben im Altertum,I,p.124. - Erman,A. \& Grapow,H.(1972).Wörterbuch der ägyptischen Sprache I,p.329.

- Erman,A. \& Grapow,H.(1972).Wörterbuch der ägyptischen Sprache II,p.184,2.

- Erman,A. \& Grapow,H.(1972).Wörterbuch der ägyptischen Sprache IV, p.473(15).

- Fakhry, A.( 1952). The Inscriptions of the Amethyst Quarries at Wadi El Hudi, Cairo, p.33 fig. 27,p.44 n 228 fig. 36.

Faulkner,R.(1981). A Concise Dictionary of Middle Egyptian, Oxford, p.64, p.123.

- Franke, D.( 1983). Altagyptische Verwandtsbezeichnungen im Mittleren Reich, Hamburg,p. 21.

-Gardiner, A.H. (1931). The Chester Beatty Papyri, No 1, p.14 photnote 3. Hayes,W.C. (1953). The Scepter of Egypt, New York, p.346.

- Helck, W. (1954). <<Untersuchungen zu den Beamtentiteln des Agyptischen Alten Reiches〉>, Agyptologische Forschungen 18, p.19.

-Helck, W.( 1987). <<Untersuchungen zur Thinitenzeit〉>, Wiesbaden, Agyptologische Abhandlungen 45,p.243.

- Jones, D.( 2000). An Index of Ancient Egyptian Titles, Epithets and Phrases of Old Kingdom I, International Series 866, p.388 n 1437.

${ }^{58}$ Junker, H.( 1939). <<PHr nfr〉>, ZÄS 75, Leipzig,p.71. Dans Pyr,W.285 et Berlin 1154,34. 
Junker, H. ( 1939). <<PHr nfr〉>, Zeitschrift für Ägyptische Sprache und Altertumskunde

75, Leipzig, p.71.

- Martin, G.T.( 1979). <<Private-name Seals in the Alnwick Castle

Collection $>$, Mitteilungen Deutschen Archaologischen Instituto

Abteilungen 35, Meinz, p.218 n 17.

- Meulenaere, H.de.( 1981). <<Contributions à la prosopographie du Moyen

Empire >, supp. Bulletin de l'Institut Français d'Archéologie Orientale 81,

Le Caire,p. 81

Meulenaere, De. ( 1977). <<Review of W.K.Simpson, The Terrace of the

Great God at Abydos, The Offering Chapels of Dynasties 12 and 13>>,

Chronique d'Egypte 52 n 103, Bruxelles, p.8.

- Murray,A.( 2004) Index of Names and Titles of The Old Kingdom, New York, p.XIX.

-Pirenne, J. (1934).Histoire des Institutions et du droit privé de l'ancienne

Egypte II, Bruxelles, pp.101-104.

- Pirenne, J.( 1935).Histoire des Institutions et du droit privé de l'ancienne Egypte III, Bruxelles pp. 73-75.

-Quirke, S. (1986). <<The Regular Titles of The Late Middle Kingdom>>, Revue d' Egyptologie 37, Louvain, p.120.

-Quirke, (2004).S. Titles and Bureaux of Egypt, Golden House Publication, Egyptology I, London, p.87.

Rowe, A. (1939). <<Three new Stelae from the south Eastern Desert>>, Annales du Service des Antiquités Egyptiennes 39, Le Caire, p. 192 photenote n 1.

-Sethe, K.(1907-8). <<Die Namen von Ober und Unteragypten usw〉>, Zeitschrift für Ägyptische Sprache und Altertumskunde 44, Leipzig, pp.1516.

Sethe, K. ( 1918). $<<Z u$ den mit wr $>>$ der größe $<<$ beginnenden alten Titeln〉>, Zeitschrift für Ägyptische Sprache und Altertumskunde 55, Leipzig, p.66.

-Spalinger, A.( 1980). $<<$ Remarks on the Family of Queen x a-s-nbw and the Problem of Kingship in Dynasty XIII >>, Revue d'Egyptologie 32, Louvain, p.97.

-Stefanovic, D.( 2009). The Non-Royal Regular Feminine Titles of the Middle Kingdom and Second intermediate Period, Golden House Publication, London, p.35.

-Strudwick, N.( 1985). The Administration of Egypt in the Old Kingdom, the highest titles and Their Holders, Boston, pp. p.179 n

36,31,91,124,132,87,99;197,222.

Vernus, P. (1986). Le surnom au Moyen Empire,Rome, p.12 n 47,p.21 n 89, p. 27 n 114,p.37 n 160, p.56 n 261,p.57 n 261, p.71 n 323, p.72 n 330. 
- Vernus, P.( 1974).<<Un Pseudo-Naos de la XIII dynastie>>, Revue d'Egyptologie 26, Paris, pp.107,109,111.

Walle, B.Van de \& Meulenaere, H.de.( 1973). <<Compléments à la prosopographie médicale >, Revue d'Egyptologie 25, Paris, p.71.

-Walle, Van de. (1938). <<Un hymne du Moyen Empire complété à l'aide de deux stèles du Musée National de Rio de Janero〉>, Revue d'Egyptologie 3, Le Caire, p.96.

Ward, W.( 1986). Essays on Feminine Titles of the Middle Kingdom, and Related Subjects, Beirut, pp.120,123.

- Ward, W. (1982). Index of Egyptian Administrative and Religious Titles in The Middle Kingdom with a Glossary of Words and Phrases used by W.Ward, Beirut, p.87 n 721. 